\title{
TOPOLOGICAL EQUIVALENCE IN THE SPACE OF INTEGRABLE VECTOR-VALUED FUNCTIONS
}

\author{
SEMION GUTMAN
}

\begin{abstract}
The Banach space $L^{1}(0, T ; X)$ is retopologized by $\|f\|\|=\max \| \int_{a}^{b} f d t \|$, $0 \leqslant a \leqslant b \leqslant T$, where $\|\cdot\|$ is the norm in the given Banach space $X$. It is shown here that this topology coincides with the usual weak topology of $L^{1}(0, T ; X)$ on a wide class of weakly compact subsets.
\end{abstract}

Let $X$ be a Banach space and $T>0$. Denote by $L^{1}(0, T ; X)$ the Banach space of all (Bochner) integrable functions (equivalence classes) on $[0, T]$ with the norm

$$
|f|_{1}=\int_{0}^{T}\|f(\tau)\| d \tau
$$

where $\|\cdot\|$ is the norm in $X . L^{1}(0, T ; X)$ can be retopologized with the weaker norm

$$
\|f \mid\|=\max _{0 \leqslant a \leqslant b \leqslant T}\left\|\int_{a}^{b} f(\tau) d \tau\right\| .
$$

Denote this space by $L^{1}(|\|| \cdot||)$. Note that

$$
\|f\|_{1}=\max _{0 \leqslant a \leqslant T}\left\|\int_{0}^{a} f(\tau) d \tau\right\|
$$

is an equivalent norm in $L^{1}(\||\cdot|\|)$. The space $L^{1}(\|\| \cdot \| \mid)$ was recently used to obtain existence results for some kinds of abstract differential equations (see e.g. [4], [5]). It was observed that subsets of the form

$$
\left\{f \in L^{1}(0, T ; X): f(t) \in K \text { almost everywhere on }[0, T]\right\}
$$

are compact in $L^{1}(\||\cdot|\|)$ if the set $K$ is compact in $X$. The main purpose of this note is to show that for a wide class of subsets of $L^{1}(0, T ; X)$ (particularly of the above type), the topology generated by $\||\cdot|\|$ coincides with the usual weak topology of $L^{1}(0, T ; X)$. On the connections between $L^{1}(0, T ; H)$ and $L^{1}(\||\cdot| \mid)$, where $H$ is a Hilbert space, see [5].

Definition. We say that a set $F \subset L^{1}(0, T ; X)$ has property (U) if:

(i) $F$ is bounded and uniformly integrable.

(ii) For every $\varepsilon>0$ there exists a compact set $K_{\varepsilon} \subset X$ such that for every $f \in F$ there exists a measurable set $\Omega_{f, \varepsilon}$ with $\mu\left([0, T] \backslash \Omega_{f, \varepsilon}\right) \leqslant \varepsilon$ and $f(t) \in K_{\varepsilon}$ for $t \in \Omega_{f, \varepsilon^{*}}$

Received by the editors November 1, 1983, and, in revised form, February 23, 1984.

1980 Mathematics Subject Classification. Primary 46E40, 28B05.

Key words and phrases. Vector-valued functions, weak compactness, Banach spaces, topological equivalence. 
Here $\mu$ is the Lebesgue measure on [0,T]. On the property (U), see [1 and 3].

Recall that if we denote by $l_{1}$ the Banach space of all real summable sequences $\left\{\xi_{i}\right\}_{1}^{\infty}$ with norm $|x|_{1}=\sum_{1}^{\infty}\left|\xi_{i}\right|$, where $x=\left\{\xi_{i}\right\}_{1}^{\infty} \in l_{1}$, then the conjugate space $m$ is the space of all real bounded sequences $\left\{\zeta_{i}\right\}_{1}^{\infty}$ with norm $|y|_{\infty}=\sup _{i}\left|\zeta_{i}\right|$, where $y=\left\{\zeta_{i}\right\}_{1}^{\infty} \in m$. Let $K$ be a compact set. Denote by $C(K)$ the Banach space of all continuous functions on $K$ with sup-norm $|\cdot|_{\infty}$. The conjugate $(C(K))^{*}=M(K)$ is the Banach space of all Radon measures on $K$. The conjugate $\left(L^{1}(0, T ; X)\right)^{*}$ is the space $\Lambda\left(0, T ; X^{*}\right)$ of all essentially bounded scalar measurable functions $g:[0, T] \rightarrow$ $X^{*}$, and every linear continuous functional on $L^{1}(0, T ; X)$ is given by

$$
f \rightarrow \int_{0}^{T}(f(\tau), g(\tau)) d \tau
$$

where $f \in L^{1}(0, T ; X), g \in \Lambda\left(0, T ; X^{*}\right)$ and $(\cdot, \cdot)$ is the pairing between $X$ and $X^{*}$. (See [2, 8.14-8.18].) Our main result is the following:

TheOREM. Let $X$ be a Banach space and $T>0$. Let the set $F \subset L^{1}(0, T ; X)$ have property (U). Then the weak topology of $L^{1}(0, T ; X)$ and the topology of $L^{1}(\|\| \cdot \|)$ coincide on $F$. Moreover, $F$ is relatively compact in $L^{1}(||||||)$.

Proof. Note that the set $\bigcup_{n=1}^{\infty} K_{1 / n}$ is separable in $X\left(K_{1 / n}\right.$ is defined as in the Definition). Therefore $F$ is separable in $L^{1}(0, T ; X)$ and we can suppose without loss of generality, that $X$ is separable.

Let $\left\{t_{n}\right\}_{1}^{\infty}$ be a dense sequence in $[0, T]$ and $\left\{y_{n}^{*}\right\}_{1}^{\infty}$ a weak-star dense sequence in the unit ball $S^{*}$ of $X^{*}$. Let $\chi\left[t_{n}, t_{m}\right]$ be a characteristic function of the interval $\left[t_{n}, t_{m}\right]$. Then the set of all the functions of the form $y_{n}^{*} \cdot \chi\left[t_{p}, t_{q}\right], t_{p}<t_{q}$, is countable. Denote these functions by $\left\{\phi_{n}\right\}_{1}^{\infty}$. Then

$$
\|f\|=\sup _{n}\left|\int_{0}^{T}\left(f(\tau), \phi_{n}(\tau)\right) d \tau\right| .
$$

Define a linear continuous operator $P: L^{1}(0, T ; X) \rightarrow m$ by

$$
P f=\left\{\int_{0}^{T}\left(f(\tau), \phi_{n}(\tau)\right) d \tau\right\}_{n=1}^{\infty} .
$$

Note that $\left|\left\|f|\||=|P f|_{\infty}\right.\right.$.

We will prove that $P(F) \subset m$ is relatively (norm) compact in $m$. Suppose there exists a compact set $K \subset X$ such that $F \subset F(K)=\left\{f \in L^{1}(0, T ; X): f(\tau) \in K\right.$ almost everywhere on $[0, T]\}$. Recall that $C(K)$ is the Banach space of continuous functions on $K$, and define the operator $\hat{P}: l_{1} \rightarrow L^{1}(0, T ; C(K))$ by $\hat{P} e_{n}=\phi_{n}$ on the standard basis $\left\{e_{n}\right\}_{1}^{\infty}$ of $l_{1}$, and then extend it by linearity and continuity to all $l_{1}$. The set $\left\{\phi_{n}\right\}_{1}^{\infty} \subset L^{1}(0, T ; C(K))$ is norm compact. This can be checked directly or by using the criterion of compactness in the spaces $L^{P}(0, T ; X)$ (see [3, Theorem A.1]). Thus $\hat{P}$ is compact. Its dual, $\hat{P}^{*}:\left(L^{1}(0, T ; C(K))\right)^{*} \rightarrow m$, is also compact. Thus $\hat{P}^{*}: \Lambda(0, T ; M(K)) \rightarrow m$,

$$
P^{*} g=\left\{\int_{0}^{T}\left(\phi_{n}(\tau), g(\tau)\right) d \tau\right\}_{n=1}^{\infty}
$$


where the pairing $(\cdot, \cdot)$ is

$$
\left(\phi_{n}(\tau), g(\tau)\right)=\int_{K} \phi_{n}(x, \tau) d g(x, \tau)
$$

for the measure $d g(x, \tau)$ corresponding to $g(\tau)$. In particular, if $g(\tau)$ is a Dirac measure for each $\tau \in[0, T]$ and $g(\tau)$ is concentrated at $f(\tau) \in K \subset X$, then $g \in \Lambda(0, T ; M(K))$ and

$$
\hat{P}^{*} g=\left\{\int_{0}^{T}\left(f(\tau), \phi_{n}(\tau)\right) d \tau\right\}_{n=1}^{\infty}=P f .
$$

Thus the action of the operator $\hat{P}$ on $F$ can be identified with the action of the operator $\hat{P}^{*}$, and the image $P(F)$ is relatively compact in $m$. Now we can suppose that $F$ is a general set with property (U).

Consider the sets $F_{\varepsilon}=\left\{f \cdot \chi\left(\Omega_{f, \varepsilon}\right): f \in F\right\}$. Here $\Omega_{f, \varepsilon}$ is a measurable set as in the Definition and $\chi\left(\Omega_{f, \varepsilon}\right)$ is its characteristic function. The set $F$ is uniformly integrable, hence for each $\delta>0$ there exists an $\varepsilon>0$ such that $\inf \left\{|f-g|_{1}: g \in F_{\varepsilon}\right\} \leqslant \delta$ for every $f \in F$. Note that $\||h|\| \leqslant|h|_{1}$ for each $h \in L^{1}(0, T ; X)$. Therefore, by definition of $P$, we have $\inf \left\{|P f-y|_{\infty}: y \in P F_{\varepsilon}\right\} \leqslant \delta$. But any set $P F_{\varepsilon}$ is relatively compact in $m$, hence the set $P F \subset m$ is relatively compact. Since $P: L^{1}(0, T ; X) \rightarrow m$ is continuous in the norm topologies, it is also continuous in the weak topologies. The weak and strong topologies coincide on $P F$. Thus the restriction $\left.P\right|_{F}$ is continuous if we take the weak topology in $L^{1}(0, T ; X)$ and the strong one in $m$. By [1, Proposition 13] any set $F$ with property (U) is relatively weakly compact in $L^{1}(0, T ; X)$. Since we can suppose that $F$ is convex, the linear map $P: F \rightarrow P F$ is a homeomorphism in these topologies. The strong topology of $m$ on $P F$ is the strong topology of $L^{1}(\||\cdot|\|)$ on $F$. Thus $F$ is relatively compact in $L^{1}(\|\| \cdot \|)$ and the theorem is proved.

ACKNOWLEDGEMENT. The author is very grateful to the referee for his valuable suggestions.

\section{REFERENCES}

1. J. Bourgain, An averaging result for $l_{1}$-sequences and application to weakly conditionally compact sets in $L_{X}^{1}$, Israel J. Math. 32 (1979), 289-298.

2. R. E. Edwards, Functional analysis, Holt, Rinehart and Winston, New York, 1965.

3. S. Gutman, Compact perturbations of m-accretive operators in general Banach spaces, SIAM J. Math. Anal. 13 (1982), 789-800.

4. E. Schechter, Evolution generated by continuous dissipative plus compact operators, Bull. London. Math. Soc. 13 (1981), 303-308.

5. ___ Perturbations of regularizing maximal monotone operators, Israel J. Math. 43 (1982), 49-61.

Department of Mathematics, North Carolina State University, Raleigh, North Carolina 27650

Current address: Department of Mathematics, Vanderbilt University, Nashville, Tennessee 37235 